\title{
Escalas de avaliação do comportamento alimentar de indivíduos com transtorno do espectro autista
}

\section{Cristiane Pinheiro Lázaro' \\ Escola Bahiana de Medicina e Saúde Pública - BAHIANA, BA, Brasil}

Jean Caron

McGill University, Douglas Mental Health University Institute, Montreal, Quebec, Canadá

Milena Pereira Pondé

Escola Bahiana de Medicina e Saúde Pública, BAHIANA, BA, Brasil

\begin{abstract}
Resumo: $O$ transtorno do espectro autista (TEA) é caracterizado por déficits na interação social, comunicação verbal e não verbal, associados a padrões de comportamento repetitivos, estereotipados e interesses restritos nos comportamentos. Problemas comportamentais durante as refeições relacionados à recusa e seletividade alimentar são observados e impactam negativamente a saúde dessa população. Foi feita uma revisão sistemática dos instrumentos de avaliação disponíveis na literatura. Realizou-se busca no PubMed, Cochrane, SciELO, Lilacs, Science Direct e Embase com a combinação das palavras-chave: autismo, transtorno do espectro autista, distúrbios alimentares, comer seletivo, comportamento alimentar, seletividade alimentar, problemas de alimentação, distúrbios do processamento sensorial, questionário, avaliação, escala, inventário, ferramenta de rastreio. Foram encontrados 52 estudos, dos quais cinco satisfizeram os critérios de inclusão. Após a revisão, apontaram-se as limitações metodológicas nos trabalhos publicados e a necessidade de elaborar novo instrumento que contemple as impressões dos pais e a gravidade do transtorno.
\end{abstract}

Palavras-chave: transtorno do espectro autista; escala; transtorno da alimentação; comportamento alimentar; TEA.

\section{SCALES ASSESSING EATING BEHAVIOR IN AUTISM SPECTRUM DISORDER}

\begin{abstract}
Autism Spectrum Disorder (ASD) is characterized by impaired social interaction, impaired verbal and nonverbal communication, and repetitive, stereotyped, and restricted patterns of behavior and interests. Mealtime behavioral problems related to food refusal and selectivity were found to affect the health of this population negatively. A systematic literature review was conducted regarding existing evaluation instruments by searching PubMed, Cochrane, SciELO, Lilacs, Science Direct, and Embase databases using combinations of the following keywords: autism, autism spectrum disorder, eating disorders, selective eating, eating behavior, food selectivity, eating problems, sensory processing disorders, questionnaire, evaluation, scale, inventory, and screening tool. Fifty-two studies were found, five of which met the inclusion criteria. The review identified significant methodological limitations in the studies and revealed the need for a new instrument to take the parents' views and the severity of the disorder into consideration.
\end{abstract}

Keywords: Autism Spectrum Disorder; scales; eating disorders; feeding behavior; ASD.

${ }^{1}$ Endereço de correspondência: Cristiane Pinheiro Lázaro: Laboratório Interdisciplinar de Pesquisa em Autismo (LABIRINTO), Avenida Dom João VI, 275, Brotas, Salvador, BA. CEP: 40290-000. E-mail: lazarocris@hotmail.com 


\begin{abstract}
Resumen: El trastorno del espectro autista (TEA) se caracteriza por déficits en la interacción social, la comunicación verbal y no verbal, por un perfil de patrones de comportamiento repetitivos, estereotipados e intereses restringidos, y problemas de comportamiento durante las comidas, relacionados con la selectividad alimentaria. Para explorar el comportamiento alimentario en TEA se desarrolló una revisión sistemática de los instrumentos de evaluación en la literatura. Se realizó una búsqueda en PubMed, Cochrane, SciELO, Lilacs, Science Direct y Embase con la combinación de palabras clave: autismo, trastorno del espectro autista, trastornos de la alimentación, capricho para comer, comer, la selectividad de alimentos, problemas de alimentación, trastornos el procesamiento sensorial, cuestionario, la evaluación, la escala, la herramienta de seguimiento de inventario. Se encontraron 52 estudios de los cuales cinco satisfacían los criterios de inclusión, sin embargo, se encontraron limitaciones metodológicas en la obra publicada y la necesidad de desarrollar un nuevo instrumento.
\end{abstract}

Palabras clave: trastorno del espectro autista; escala; trastornos de la alimentación; comportamiento alimentario; TEA.

\title{
Introdução
}

Em crianças diagnosticadas com o transtorno do espectro autista (TEA), são observados sintomas relacionados a déficit na interação social recíproca e na comunicação verbal e não verbal, assim como padrões repetitivos, estereotipados e interesses restritos nos comportamentos (American Psychiatric Association, 2013). Outras características comportamentais, a exemplo de ansiedade, reações agressivas e obsessivo-compulsivas, hiperatividade, déficit de atenção e distúrbios do sono, também são frequentemente relatadas nesses indivíduos (Novaes, Pondé, \& Freire, 2008). Além dessa sintomatologia, pais e cuidadores de crianças com TEA relatam hábitos peculiares relacionados aos alimentos e ao ato comer (Lockner, Crowe, \& Skipper, 2008). Fatores intrínsecos aos alimentos podem interferir no comportamento alimentar, a exemplo de textura, cor, sabor, forma, temperatura dos alimentos, bem como o formato e a cor da embalagem, a apresentação do prato e utensílios utilizados (Mari-Bauset, Zazpe, Mari-Sanchis, Llopis-Gonzalez, \& Morales-Suarez-Varela, 2013).

Em paralelo às caraterísticas do transtorno, crianças com desenvolvimento típico na faixa etária dos 18 aos 24 meses tendem a apresentar dificuldades na aceitação de novos sabores, o que pode originar um consumo limitado e inadequado dos alimentos (Johnson, 2016). Esse comportamento caracterizado pela neofobia - que faz parte do desenvolvimento infantil típico - poderá ser exacerbado no contexto de comportamento restritivo de TEA.

A recusa de determinadas texturas e de provar novos alimentos influencia significativamente na formação dos hábitos alimentares das crianças com TEA (Bandini et al., 2010), contribuindo para um comer seletivo. A seletividade alimentar pode se manifestar na recusa de determinados alimentos, em um repertório limitado de opções, ou ainda na alta frequência na ingestão de um único alimento (Bandini et al., 2010). Não existe um consenso quanto à classificação da seletividade alimentar, mas os problemas relacionados à ingestão de alimentos variam desde casos leves, em que o comporta- 
mento não representa um risco para a saúde, até mais graves, que levam ao risco de desnutrição (Hyman et al., 2012). Além dos fatores intrínsecos aos alimentos, no TEA é comum observar um grave transtorno alimentar denominado pica, que consiste na ingestão recorrente de substâncias e objetos estranhos que não são considerados alimentos (tinta, giz, papel, terra, carvão, entre outros) (Call, Simmons, Mevers, \& Alvarez, 2015).

Pesquisas que investigaram especificamente os problemas alimentares em pessoas com TEA indicam que uma parcela dessa população também apresenta dificuldades motoras orais relacionadas à mastigação e à deglutição (Sacrey, Germani, Bryson, \& Zwaigenbaum, 2014), problemas no trato gastrointestinal (TGI) (Chaidez, Hansen, \& Hertz-Picciotto, 2014) e disfunção sensorial (Cermak, Curtin, \& Bandini, 2010; Green \& Ben-Sasson, 2010). Os problemas relacionados à modulação sensorial, tanto na forma hipo quanto hiper-reativa, interferem diretamente no paladar, olfato, audição, visão, tato, sistema vestibular e propriocepção (Green \& Ben-Sasson, 2010). Assim, as alterações sensoriais, musculares e gastrointestinais podem influenciar de forma direta ou indireta a alimentação. Além disso, a seletividade alimentar pode estar associada aos interesses rígidos e restritos característicos do comportamento autista. A investigação das particularidades do comportamento alimentar das pessoas com TEA pode ser mais bem investigada e classificada a partir de instrumentos de avaliação estruturados, que contemplem as diversidades dos possíveis comportamentos.

A necessidade de investigar melhor o comportamento alimentar dos indivíduos com TEA levou alguns pesquisadores a elaborar instrumentos de avaliação que pudessem ser preenchidos pelos pais ou cuidadores, buscando identificar informações relativas ao comportamento alimentar e a outras variáveis que pudessem influenciar a alimentação. Este estudo tem por objetivo realizar uma revisão sistemática dos instrumentos disponíveis na literatura que avaliam o comportamento alimentar de indivíduos com TEA.

\section{Método}

\section{Estratégia para busca de dados}

Foi realizada busca em periódicos científicos nacionais e internacionais até o ano de 2015, sem data inicial específica. O processo de busca dos artigos para a inclusão nesta revisão foi realizado com a combinação das seguintes palavras-chave: autismo, transtorno do espectro do autismo, transtorno alimentar, alimentação seletiva, comportamento alimentar, seletividade alimentar, problema alimentar, transtorno do processamento sensorial, questionário, avaliação, escala, inventário, ferramentas de avaliação, autism, autism spectrum disorder, eating disorders, selective eating, food behavior, food selectivity, feeding problems, sensory processing disorders, questionnaire, assessment, scale, inventory, screening tool. Os descritores foram escolhidos por pesquisadores com ampla experiência no tratamento do TEA. A busca foi realizada nas seguintes bases de dados: PubMed, Cochrane, SciELO, Lilacs, Science Direct e 
Embase, Google acadêmico, literatura cinzenta (Capes: http://bancodeteses.capes.gov. br/banco-teses/\#/; The New York Academy of Medicine: http://www.nyam.org/library/ collections-and-resources/grey-literature-report/).

\section{Critério de eleição}

Os seguintes critérios de elegibilidade foram aplicados: 1. artigos publicados em inglês, português ou espanhol e 2. instrumentos elaborados com o objetivo de avaliar os problemas relacionados ao comportamento alimentar em indivíduos com o TEA. Estudos em outras línguas foram excluídos. Extraíram-se as seguintes informações de artigos elegíveis: 1. faixa etária, 2. objetivo do estudo e 3. variáveis avaliadas.

Um dos pesquisadores avaliou os títulos, resumos ou ambos os resultados obtidos a partir dos bancos de dados.

\section{Resultados}

O rastreamento segundo os critérios estabelecidos resultou em 52 artigos (Figura 1), dos quais 40 foram excluídos por não preencherem os critérios de inclusão ou por estarem repetidos. Dos 12 artigos potencialmente escolhidos na triagem do título e resumo, sete foram excluídos por não serem instrumentos de avaliação. Dos cinco artigos incluídos três foram realizados nos Estados Unidos, um na Suécia e um no Canadá, incluindo crianças, jovens e adultos com TEA na faixa etária de 3 a 25 anos. Desses artigos, um foi eliminado por não ter como foco da investigação o TEA.

\section{Figura I. Resultado da pesquisa bibliográfica nas bases de dados eletrônicas.}

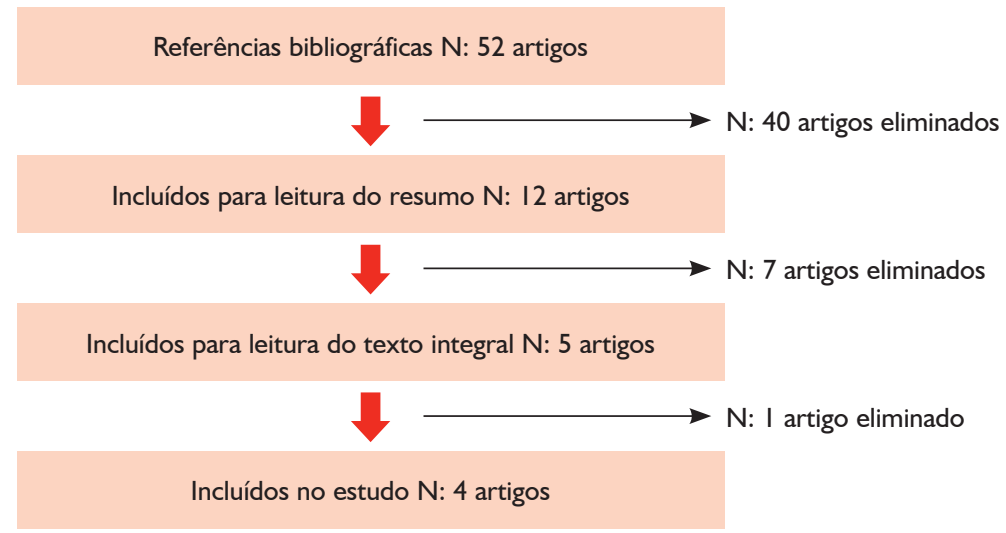

As características dos estudos incluídos nesta revisão estão listadas no Quadro 1 e descritas a seguir. 


\section{Quadro I. Característica dos estudos incluídos na revisão}

\begin{tabular}{|c|c|c|c|c|}
\hline Autor & Instrumento & N/ faixa etária & Objetivo & Fatores avaliados \\
\hline $\begin{array}{l}\text { Lukens \& } \\
\text { Linscheid } \\
\text { (2008) } \\
\text { EUA }\end{array}$ & $\begin{array}{l}\text { Brief Autism } \\
\text { Mealtime Behavior } \\
\text { Inventory (BAMBI) }\end{array}$ & $\begin{array}{l}40 \text { crianças típicas } \\
\text { e } 68 \text { TEA } \\
\text { 3-II anos }\end{array}$ & $\begin{array}{l}\text { Desenvolver uma } \\
\text { medida para avaliar } \\
\text { os problemas } \\
\text { comportamentais } \\
\text { das refeições em } \\
\text { crianças com TEA. }\end{array}$ & $\begin{array}{l}\text { Comportamento } \\
\text { disruptivo, recusa } \\
\text { alimentar, } \\
\text { comportamentos } \\
\text { ritualísticos e } \\
\text { estereotipados, } \\
\text { preferências alimentares } \\
\text { restritas, variedade } \\
\text { limitada de alimentos. } \\
\text { Questionário eletrônico } \\
\text { contendo } 20 \text { itens. }\end{array}$ \\
\hline $\begin{array}{l}\text { Seiverling, } \\
\text { Hendy, \& } \\
\text { Williams } \\
(20 \mathrm{I} \text { I) } \\
\text { EUA }\end{array}$ & $\begin{array}{l}\text { Screening Tool for } \\
\text { Feeding Problems } \\
\text { (STEP-CHILD) }\end{array}$ & $\begin{array}{l}\text { I } 42 \text { crianças } \\
\text { ( } 95 \text { meninos, } \\
47 \text { meninas; } 43 \text { com } \\
\text { TEA, } 5 \text { I com outras } \\
\text { necessidades } \\
\text { especiais, } 48 \text { com } \\
\text { desenvolvimento } \\
\text { típico e com } \\
\text { problemas } \\
\text { alimentares) }\end{array}$ & $\begin{array}{l}\text { Avaliar se os } \\
23 \text { itens do STEP } \\
\text { proposto por } \\
\text { Matson \& Kuhn } \\
\text { (200I) poderiam } \\
\text { ser usados como } \\
\text { uma medida de } \\
\text { problemas ligados } \\
\text { à alimentação em } \\
\text { uma amostra de } \\
\text { crianças. }\end{array}$ & $\begin{array}{l}\text { Problemas de } \\
\text { mastigação, comer } \\
\text { rápido, recusa de } \\
\text { alimento, seletividade } \\
\text { alimentar, vômitos e } \\
\text { roubar comida. }\end{array}$ \\
\hline $\begin{array}{l}\text { Karlsson, } \\
\text { Rastam, \& } \\
\text { Went (20I3) } \\
\text { Suécia }\end{array}$ & $\begin{array}{l}\text { SWedish Eating } \\
\text { Assessment for } \\
\text { Autism Spectrum } \\
\text { Disorder (SWEAA) }\end{array}$ & $\begin{array}{l}57 \text { TEA e } \\
31 \text { controles } \\
15-25 \text { anos }\end{array}$ & $\begin{array}{l}\text { Elaborar e validar } \\
\text { um questionário } \\
\text { referente aos } \\
\text { problemas } \\
\text { alimentares em } \\
\text { indivíduos com } \\
\text { inteligência normal, } \\
\text { dentro do TEA. }\end{array}$ & $\begin{array}{l}\text { Percepção, controle } \\
\text { motor, comportamento } \\
\text { alimentar, situação social } \\
\text { nas refeições, outros } \\
\text { comportamentos } \\
\text { associados com } \\
\text { distúrbio alimentar, } \\
\text { fome/saciedade, } \\
\text { capacidade simultânea, } \\
\text { pica, ambiente das } \\
\text { refeições, capacidade } \\
\text { simultânea. }\end{array}$ \\
\hline $\begin{array}{l}\text { Allen et al. } \\
(20 \mid 5) \\
\text { Canadá }\end{array}$ & $\begin{array}{l}\text { Behavioral Pediatrics } \\
\text { Feeding Assessment } \\
\text { Scale (BPFAS) }\end{array}$ & $\begin{array}{l}347 \text { crianças } \\
\text { com TEA } \\
2-5 \text { anos de idade }\end{array}$ & $\begin{array}{l}\text { Verificar se o } \\
\text { BPFAS é capaz } \\
\text { de avaliar os } \\
\text { problemas } \\
\text { alimentares no } \\
\text { TEA. }\end{array}$ & $\begin{array}{l}\text { Seletividade alimentar, } \\
\text { recusa geral, recusa de } \\
\text { alimentos com textura, } \\
\text { recusa por crianças mais } \\
\text { velhas, crianças que } \\
\text { demoram em comer ou } \\
\text { "ficam enrolando" }\end{array}$ \\
\hline
\end{tabular}

* TEA: transtorno do espectro autista; STEP: Screening Tool for Feeding Problems; pica: ingerir coisas que não são consideradas alimento. 


\section{Brief Autism Mealtime Behavior Inventory (BAMBI)}

Lukens e Linscheid (2008) foram os primeiros a desenvolver uma escala com o objetivo de avaliar a natureza dos problemas relacionados ao comportamento alimentar de crianças com autismo, chamada Brief Autism Mealtime Behavior Inventory (BAMBI). Para o estudo, a amostra foi composta por 68 crianças com TEA e 40 com desenvolvimento típico, com idade entre 3 e 11 anos. Foi solicitado aos cuidadores dessas crianças que preenchessem, por meio de uma página na internet, o BAMBI; o Gilliam Autism Rating Scale (GARS) (Gilliam, 1995), que é uma escala que avalia a frequência e a severidade dos comportamentos observados no TEA; o Behavioral Pediatric Feeding Assessment Scale (BPFAS) (Crist, \& Napier-Phillips, 2001), que avalia o comportamento alimentar de crianças; o questionário de frequência alimentar Youth/Adolescent Questionnaire (YAQ) (Rockett et al., 1997) e o recordatório de 24 horas com o objetivo de estimar o consumo médio dos alimentos.

A escala BAMBI foi construída a partir da literatura que descreve e avalia intervenções pediátricas para crianças autistas com problemas alimentares. A escala continha inicialmente 20 itens sobre o comportamento da criança, que deveriam ser preenchidos pelos cuidadores. Cada item continha cinco opções de resposta que variavam de "nunca" a "sempre". Os escores mais elevados indicavam problema mais grave. Os itens dessa escala eram os seguintes: chora ou grita durante as refeições; vira o rosto ou o corpo para o lado oposto ao da comida; expele o alimento que comeu; tem comportamento disruptivo durante as refeições; fecha a boca com força quando a comida é apresentada; fica sentado à mesa até a comida acabar; é agressivo durante as refeições; tem comportamento autolesivo durante as refeições; é flexível no que se refere à rotina das refeições; recusa-se a comer alimentos que exijam muita mastigação; fica na expectativa de provar novos alimentos; não gosta de determinados alimentos e não os ingere; prefere repetir a mesma comida em todas as refeições; prefere alimentos crocantes; aceita ou prefere alimentos variados; prefere que a comida seja servida de uma forma determinada; prefere apenas alimentos doces; prefere a comida preparada de um modo particular.

A versão preliminar com os 20 itens foi submetida à análise psicométrica em um estudo-piloto com 50 participantes (Lukens, 2002). Essa análise indicou um coeficiente de 0,61 na escala total, e a análise fatorial indicou três fatores responsáveis por $45 \%$ da variância no escore total do problema comportamental investigado. Nove itens se relacionaram ao fator "recusa alimentar/comportamento disruptivo" e correspondiam a $20 \%$ da variância. Cinco itens se relacionaram com o fator "variedade limitada", com $18 \%$ da variância. Cinco itens se relacionaram com o terceiro fator, entretanto não puderam ser interpretados. Assim, após análises alguns itens foram removidos, gerando uma versão final da escala com18 itens.

A análise da consistência interna da versão final da escala com 18 itens demonstrou um valor de 0,88 na amostra final, sendo indicados três fatores: "variedade limitada", "recusa alimentar" e "características do autismo", que não haviam aparecido no estudo-piloto com 20 itens. 
Para avaliar a estabilidade, o BAMBI foi novamente preenchido após sete meses (intervalo: 5-13 meses) em 31\% da amostra inicial. Um coeficiente de confiabilidade teste-reteste foi calculado entre primeira e a segunda aplicação do BAMBI, apresentando valor significativo de $r(33)=0,87, p<0,01$. Na avaliação da confiabilidade interavaliadores, o comportamento alimentar avaliado pelo BAMBI e observado por professores e cuidadores demonstrou ser forte e positiva com $r(16)=0,78, p<0,01$.

O BAMBI também apresentou correlações positivas entre os fatores (variedade limitada, recusa alimentar, características do autismo) ao avaliar a validade interna. Correlações positivas também foram encontradas na validade convergente. As correlações foram calculadas entre o escore de frequência total do BAMBI e a pontuação da escala BPFAS, que avalia a frequência e a severidade dos comportamentos observados no TEA ("frequência do comportamento da criança" $r(108)=0,77, p<0,01$; "sentimentos/estratégias dos pais" $r(108)=0,74, p<0,01$; "frequência total da BFAS" $r(108)=$ $0,82, p<0,01)$.

Também foram calculadas correlações entre escores fatoriais individuais e medidas de critérios externos para verificar a validade concomitante, e os valores positivos encontrados sugerem que o BAMBI explora de forma adequada as características do autismo descritas no Manual diagnóstico e estatístico de transtornos mentais (DSM-IV).

No que diz respeito à capacidade discriminatória do $\mathrm{BAMBI}$, houve diferenças estatisticamente significativas por meio da one-way ANOVA $(p<0,01)$ entre os grupos na frequência de problemas comportamentais na hora das refeições, com escores significativamente mais elevados para as crianças com autismo do que para as crianças com desenvolvimento típico.

\section{Screening Tool for Feeding Problems (STEP-CHILD)}

Seiverling, Hendy, \& Williams (2011) desenvolveram o STEP-CHILD com o propósito de mensurar os problemas alimentares de crianças. Os autores partiram do Screening Tool of Feeding Problems (STEP) (Matson \& Kuhn, 2001), escala que avalia problemas na alimentação de adultos com deficiência intelectual. O STEP-CHILD foi aplicado em uma amostra de 142 crianças com idade média de 6,14 meses, sendo 43 crianças com TEA, 51 com outras necessidades especiais e 48 sem alterações no desenvolvimento com problemas alimentares, todas recrutadas em uma clínica especializada em problemas alimentares. Inicialmente os pais preencheram os 23 itens originais do STEP, cada item referente a uma característica da alimentação. Os itens têm uma classificação do tipo Likert de três gradações ( $0=$ ausente, $1=1$ a 10 vezes por mês, $2=$ mais de 10 vezes por mês), para indicar a frequência do comportamento apresentado por seus filhos. Em paralelo, os pais preencheram quatro itens que avaliaram problemas relacionados à textura, um inventário sobre preferências alimentares, o Child Eating Behavior Questionnaire (CEBQ) (Wardle, Guthrie, Sanderson, \& Rapoport, 2001), que avalia problemas alimentares em crianças, e o "Parent Mealtime Action Scale" (PMAS) (Hendy, Williams, Camise, Eckman, \& Hedemann, 2009), que identifica o comportamento dos 
pais e das crianças durante as refeições e a frequência com que os pais comem e servem certos alimentos (como frutas e legumes).

Na elaboração da primeira versão do STEP-CHILD, visando determinar a sua validade de conteúdo e construto, foi utilizada análise fatorial para determinar os itens e as subescalas selecionadas a partir do STEP. Para cada dimensão, cada item demonstrou um fator de carga de 0,40 e alfa de Cronbach de 0,55 ou superior. A análise fatorial revelou 15 itens distribuídos em seis dimensões: "problemas na mastigação", "comer rápido", "recusa de alimento", "seletividade alimentar", "vômitos" e "roubar alimentos". Para avaliar a confiabilidade interna, foi obtido um coeficiente médio de 0,62 do alfa de Cronbach para as seis subescalas citadas anteriormente.

Para a avaliação da validade concomitante da escala, foram feitas correlações com instrumentos já validados. A validade concomitante dos seis domínios do STEP-CHILD foi realizada por meio de regressão múltipla entre o CEBQ (Wardle et al., 2001) e a aferição dos problemas relacionados à textura. As associações encontradas nas subcategorias foram as seguintes: "problemas da mastigação" foi significativamente correlacionado com problemas ligados à textura (Beta: 0,445 ) e negativamente com frescura para comer (Beta: -0,229); "comer rápido" foi correlacionado positivamente com excessos emocionais do CEBQ (Beta: 0,467) e negativamente com a capacidade de resposta da saciedade do CEBQ (Beta: -.434); a "recusa alimentar" foi positivamente correlacionada com a frescura para comer do CEBQ (Beta: .296); a "seletividade alimentar" foi positivamente correlacionada com problemas relacionados à textura (Beta: .406) e frescura para comer do CEBQ (Beta:.403); "roubar comida" foi positivamente correlacionado com receptividade por alimentos do CEBQ (Beta: .442) e frescura para comer (Beta: .314). A subescala "vômito" não demonstrou correlação com nenhum fator.

Ainda avaliando a validade externa e de construto, realizaram-se correlações com outras medidas, sendo elas: variáveis demográficas da amostra, antropometria (cálculo do índice de massa corporal - IMC), inventário de preferência alimentar e o PMAS (Hendy et al., 2009). A associação das variáveis demográficas da amostra com o STEP-CHILD, realizada por meio da análise de covariância entre os gêneros (meninos e meninas), a idade e os três grupos diagnosticados (crianças com autismo, outras necessidades especiais e sem necessidades especiais), apresentou significância entre as subescalas "problemas na mastigação" e idade $(p=0,004)$, e as crianças mais novas apresentaram maior frequência de problemas $(p=0,008)$ e "roubar comida" associada com o diagnóstico ( $p=0,012)$. Já a correlação com a antropometria (peso, estatura e IMC) demonstrou significância apenas entre a subescala "comer rápido" e criança com alto valor de IMC $(p<0,001)$. Utilizando os dados coletados com o inventário de preferência alimentar, a correlação positiva foi observada entre a dimensão "seletividade alimentar" e a reduzida variedade de alimentos consumidos ( $p<0,001)$. Por fim, quando as ações dos pais durante as refeições mensuradas pelo PMAS (Hendy et al., 2009) foram analisadas, os resultados revelaram que as crianças que mais pontuaram 
na subescala "problemas na mastigação" tinham pais que raramente definiam limites para lanches $(p=0,003)$; crianças que apresentavam um comer rápido tinham pais que raramente insistiam para comer durante as refeições ( $p=0,004)$; crianças com mais seletividade nos alimentos tinham pais que muitas vezes preparavam refeições especiais diferentes dos demais integrantes da família e que muitas vezes ofereciam opções de alimentos da preferência da criança $(p<0,001)$.

Quanto à capacidade discriminatória do STEP-CHILD, o único dado disponível foi a correlação positiva da subescala "roubar comida" com o diagnóstico do transtorno $(p=0,012)$. Após o pareamento com um grupo de crianças que apresentavam outras necessidades especiais e outro sem necessidades especiais, foi observado que "roubar comida" estava mais associado com as crianças com TEA.

\section{The SWedish Eating Assessment (SWEAA)}

O terceiro instrumento encontrado foi o SWedish Eating Assessment (SWEAA) (Karlsson, Rastam, \& Went, 2013). Karlsson et al. (2013) construíram, com base numa revisão da literatura (Rastam, 2008) e nas próprias experiências clínicas, um questionário estruturado de autorrelato multidimensional, com 81 itens, destinado a avaliar o comportamento alimentar no TEA. Os participantes foram selecionados aleatoriamente em uma clínica neuropsiquiátrica para crianças, localizada dentro de um hospital da Suécia, com faixa etária entre 15 e 25 anos. Dos 202 pacientes contatados, 57 $(28 \%)$ (masculino = 38; feminino $=19)$ dos indivíduos responderam ao questionário e o completaram. Cada participante foi avaliado por um profissional especializado que confirmava o diagnóstico e aferia o coeficiente de inteligência (QI). Como critério de inclusão, foi estabelecido que os participantes devessem apresentar um nível de QI dentro da faixa de normalidade para que pudessem preencher sozinhos o questionário.

Utilizando a análise fatorial, juntamente com coeficiente alfa de Cronbach, construíram-se as subescalas e seus itens correspondentes. Após essas análises, a versão final permaneceu com 60 itens, compreendendo oito subescalas: "percepção", "controle motor", "compra de alimento", "comportamento alimentar", "ambiente das refeições", "situação social durante as refeições", "outros comportamentos associados s distúrbios alimentares" e "fome/saciedade", cujos itens eram avaliados por uma escala do tipo Likert com cinco pontos com opções de resposta variando de "nunca" a "sempre".

O SWEAA apresentou uma boa consistência interna, demonstrando valores acima de 0,30 para todas as subescalas e 0,40 para seis das oito subescalas: "controle motor", "compra de alimentos", "momento das refeições", "situação social na hora das refeições", "outros comportamentos associados com distúrbios alimentares", "fome/saciedade". Um coeficiente de alfa de Cronbach acima de 0,70 foi observado para todas as subescalas e 0,80 para seis subescalas: "percepção", "controle motor", "compra de alimento", "ambiente das refeições", "situação social durante as refeições", "outros comportamentos associados a distúrbios alimentares". 
Para avaliar a estabilidade temporal, o tempo médio entre as duas ocasiões para o teste-reteste foi de 34 dias com bom índice de correlação interclasses $(0,860)$, exceto a subescala "fome/saciedade". O mesmo teste revelou uma diferença na subescala "situação social durante as refeições", entretanto ela foi considerada apropriada para o SWEAA por causa da pequena diferença apresentada nos dois momentos.

Quanto à validade discriminante, a subescala "situação social durante as refeições" obteve a pontuação mais alta em todos os participantes do grupo clínico, e, após análises estatísticas, diferenças significativas entre os indivíduos com TEA e o grupo controle foram observadas não somente nessa subescala $(p<0,001)$, como também nas subescalas "ambiente das refeições" $(p<0,017)$ e "capacidade simultânea" $(p<0,001)$, sugerindo que esses itens sejam os principais discriminadores entre os grupos.

\section{Behavioral Pediatrics Feeding Assessment Scale (BPFAS)}

O Behavioral Pediatrics Feeding Assessment Scale (BPFAS) elaborado por Crist e Napier-Phillips (2001) é um instrumento amplamente utilizado para avaliar problemas alimentares e comportamentais em crianças. Allen et al. (2015) avaliaram a estrutura e a validade do BPFAS em uma amostra de 374 pré-escolares com TEA (314 do sexo masculino) com média de idade de 40,89 meses. Os participantes foram recrutados por meio de um estudo longitudinal em curso com os seguintes critérios de inclusão: 1. diagnóstico recente do TEA (prazo de quatro meses) informado pelo Autism Diagnostic Observation Schedule - ADOS (Lord et al., 2000), Autism Diagnostic Interview-Revised - ADI-R (Rutter, Le Couteur, \& Lord, 2003) e avaliação de um clínico; e 2. idade $\geq 2$ e $\leq 5$ anos e 0 meses. Como critérios de exclusão: 1. paralisia cerebral ou outra desordem neuromotora que possa interferir na avaliação do estudo, 2. anomalia genética ou cromossômica conhecida e 3. deficiência visual ou auditiva severa.

O BPFAS é composto por 35 itens; os primeiros 25 itens se concentram no comportamento da criança e os 10 últimos fornecem um índice de atitudes e estratégias dos pais sobre a alimentação em relação aos horários das refeições e problemas de alimentação. Em uma pontuação do tipo Likert que vai de 1 (nunca) até 5 (sempre), os pais indicaram a frequência do comportamento, e as maiores pontuações apontam níveis mais elevados dos problemas comportamentais durante as refeições.

Além do BPFAS, os pesquisadores utilizaram outras escalas para avaliar a associação de fatores da escala com as variáveis que envolviam o comportamento da criança e de seus cuidadores: funcionamento adaptativo (do nascimento aos 18 anos por meio dos domínios de comunicação, socialização, habilidades da vida diária e habilidades motoras) por meio do Vineland Adaptive Behavior Scales-Second Edition - VABS-II (Sparrow, Cicchetti, \& Balla, 2005); presença e intensidade de vários tipos de comportamentos restritos e repetitivos característicos do TEA com o Repetitive Behavior Scale-Revised RBS-R (Bodfish, Symons, Parker, \& Lewis, 2000); mensuração de vários sintomas, incluindo o comportamento social e traços do TEA, por meio do Social Responsiveness Scale - SRS (Constantino \& Gruber, 2005); comportamento da criança com o Child 
Behavior Checklist - CBCL (Achenbach \& Rescorla, 2000); problemas no sono mensurados pelo Children's Sleep Habits Questionnaire - CSHQ (Goodlin Jones, Sitnick, Tang, Liu, \& Anders, 2008; Owens, Spirito, \& McGuinn, 2000); estresse parental por meio do Parenting Stress Index-Short Form - PSI-SF (Abidin, 1995); os sintomas e a gravidade do TEA por meio do ADOS; o nível do desenvolvimento indexado pelo M-P-R (desenvolvimento motor, cognição e linguagem receptiva); habilidade na linguagem pela Preschool Language Scales-Fourth Edition - PLS-4 (Zimmerman, Steiner, \& Pond, 2002); e a idade da criança quando o responsável completou o BPFAS.

Quando se avaliou a consistência interna dos cinco fatores do BPFAS proposto por Crist \& Napier-Phillips (2001), a escala de forma geral ( $\alpha$ Cronbach $=0,82$ ) e três fatores demonstraram coeficientes aceitáveis: "comensal exigente" ( $\alpha$ Cronbach $=0,80)$, "recusa geral da consulta" ( $\alpha$ Cronbach $=0,70$ ), "crianças que postergam o momento de comer" $(\alpha$ Cronbach $=0,75)$, em contraste com "recusa de alimentos com textura" $(\alpha$ Cronbach $=$ $0,26)$, "recusa geral da criança mais velha" ( $\alpha$ Cronbach $=0,3$ ).

Quanto à validade do construto, após análise fatorial dos itens da escala, três fatores representaram $43,13 \%$ da variância e foram rotulados de "aceitação dos alimentos" ( $\alpha$ Cronbach $=0,71)$, "questões motoras orais" ( $\alpha$ Cronbach $=0,71)$ e "comportamento durante as refeições" ( $\alpha$ Cronbach $=0,81$ ).

Para avaliar a validade externa, as consequências do construto e a validade convergente, foram identificadas várias correlações positivas, variando o efeito de pequeno a médio, entre as variáveis de interesse envolvendo as crianças, seus pais, os sintomas do autismo com a aplicação de outras escalas psicométricas e os três novos fatores anteriormente mencionados.

O fator "comportamento durante as refeições" foi o que apresentou seis correlações positivas de médio efeito: os pais que relataram níveis mais elevados de sintomas de autismo pontuado pelo SRS e RBS-R (por exemplo, dificuldades sociais e de comunicação, comportamento repetitivo ou restrito) afirmaram ter mais dificuldades relacionadas com a alimentação ( $r=0,34 ; 0,40$ respectivamente). Em relação ao comportamento da criança avaliado pelo $\mathrm{CBCL}$ (internalização e externalização), foram encontradas correlações positivas médias para os fatores "questões motoras orais" ( $r=$ $0,44 ; r=0,34)$, "comportamento durante as refeições" $(r=0,47 ; r=0,48)$ e pequena correlação com "aceitação dos alimentos" ( $r=0,27 ; r=0,20)$. Assim, os pais que relataram mais problemas de comportamento tinham mais dificuldades relacionadas à alimentação. Em contrapartida, houve apenas uma pequena correlação negativa entre a gravidade do TEA indicada pelo ADOS e o fator "comportamento durante as refeições" $(r=-0,10)$, e nenhuma relação notável entre a gravidade do TEA e a "aceitação dos alimentos" $(r=0,04)$ e "questões motoras orais" $(r=-0,06)$.

As dificuldades relativas ao sono mensurado pelo CSHQ apresentaram média correlação com "comportamento durante as refeições" $(r=-0,35)$ e pequena em relação à "aceitação dos alimentos" $(r=0,19)$ e "questões motoras orais" $(r=-0,27)$. O estresse parental avaliado pelo PSI-SF demonstrou correlações positivas médias com "questões motoras orais" $(r=0,33)$ e "comportamento durante as refeições" $(r=0,38)$. Assim, os 
pais que relataram problemas frequentes relacionados à alimentação de seus filhos também relataram muitos problemas do sono com seus filhos e níveis mais elevados de estresse.

Pequenas correlações negativas entre as habilidades na vida diária e motoras pontuadas pelo VABS-II foram observadas entre "aceitação dos alimentos" $(r=-0,22)$ e "questões motoras orais" $(r=-0,11)$, sugerindo que, quanto menor o funcionamento adaptativo da criança, maiores são os problemas motores orais e de recusa alimentar.

Não foram identificadas correlações significativas entre: a idade da criança no momento em que o BPFAS foi preenchido e o funcionamento cognitivo avaliado pelo MPR em relação ao fator "aceitação dos alimentos" e "questões motoras orais". Além disso, não foram identificadas correlações significativas entre o BPFAS e a capacidade de linguagem infantil observada com o PLS-4. Contudo, pequena correlação foi observada entre o "comportamento durante as refeições" $(r=-0,19)$, idade da criança e nível cognitivo, indicando que os pais de crianças mais velhas ou mais desenvolvidas relataram níveis mais elevados de comportamento problemático nas refeições e mais dificuldades relacionadas à alimentação.

\section{Discussão}

Uma alimentação adequada na infância, desde o nascimento e durante os primeiros anos de vida, é fundamental para garantir o crescimento e o desenvolvimento normal da criança. Quando se menciona crescimento da criança, geralmente se associa ao crescimento ósseo que se reflete na estatura, mas cada um dos sistemas orgânicos também está em desenvolvimento, incluindo o sistema nervoso central. O crescimento do organismo como um todo depende de uma nutrição adequada. A nutrição e a subnutrição na primeira e na segunda fase da infância podem ter efeitos deletérios sobre o desenvolvimento infantil, assim como a ingestão em excesso que leva à obesidade (Drewett, 2010). Consequentemente, o comportamento alimentar no TEA é uma dimensão importante, pois, em muitos casos, pode colocar em risco a saúde do indivíduo. Uma propedêutica acurada do comportamento alimentar dessa população, portanto, auxilia uma possibilidade terapêutica mais individualizada e complexa. Este artigo traz uma importante contribuição ao revisar a literatura sobre os instrumentos de avaliação do comportamento alimentar de crianças com TEA, identificando limitações dos instrumentos existentes e propondo alternativas mais completas e adequadas ao diagnóstico atual do TEA.

O BAMBI (Lukens \& Linscheid, 2008) foi o primeiro instrumento desenvolvido especificamente para avaliar os problemas alimentares de crianças com autismo. A iniciativa de elaborar uma escala específica foi importante e iniciou a concepção de medidas que pudessem discriminar os problemas apresentados por autistas, comparados às crianças com desenvolvimento típico. Esse instrumento teve como foco os aspectos comportamentais relacionados à alimentação, deixando de explorar os problemas sensoriais e gastrintestinais frequentemente encontrados no TEA e que podem influenciar 
o comportamento alimentar. No processo de validação do instrumento, a amostra foi composta por apenas 68 crianças com TEA, tendo havido algumas limitações no método: o diagnóstico dos participantes não foi feito por psiquiatra, mas apenas por meio de uma escala de sintomas preenchida pelo cuidador; a investigação dos problemas alimentares não levou em conta a gravidade do TEA; a escala e os demais instrumentos de avaliação utilizados foram postados em uma página na internet sem que houvesse alguém para auxiliar em caso de dúvidas; o instrumento foi validado com uma amostra de conveniência, limitando-se às pessoas que estavam cadastradas em um site específico e que tiveram interesse em participar do estudo. Este último fato em específico demonstra significativa limitação, pois os indivíduos que optaram por não participar do estudo poderiam diferir de alguma maneira daqueles incluídos na amostra. As análises de confiabilidade revelaram alta consistência interna para os fatores "variedade limitada" e "recusa alimentar", entretanto, o fator "características do autismo" demonstrou consistência moderada. Na avaliação da estabilidade temporal, apenas menos de metade (31\%) da amostra original participou do segundo preenchimento, o que pode ter interferido no resultado. Correlações positivas entre o BAMBI e uma segunda medida de problemas no comportamento durante as refeições, previamente validada, também foram encontradas. As diferenças entre os grupos em escores totais no BAMBI apoiam a validade de construto e as correlações entre os fatores, dando suporte para a sua validade convergente. Especificamente, o fator "limitada variedade" do BAMBI foi negativamente correlacionado com porções de carne, frutas e legumes, apoiando a ideia de que crianças com TEA preferem salgadinhos e principalmente carboidratos (Ahearn, Castine, Nault, \& Green, 2001).

O STEP-CHILD (Seiverling et al., 2011), além dos aspectos comportamentais, inclui na escala itens relativos à seletividade alimentar, problemas na motricidade oral, habilidades ligadas à alimentação e recusa alimentar. Esses elementos permitiram mapear outros fatores intervenientes na construção da dinâmica alimentar de indivíduos autistas. Ambos os estudos, o BAMBI (Lukens, \& Linscheid, 2008) e o STEP-CHILD (Seiverling et al., 2011), demonstraram critérios psicométricos rigorosos ao associarem positivamente as subescalas com variáveis clínicas (peso, IMC, variedade limitada de alimentos). Além do mais, o STEP-CHILD (Seiverling et al., 2011) trouxe importante contribuição para a literatura científica ao sugerir uma associação entre variáveis ligadas à criança (peso, IMC) e aos pais (atitude dos pais durante as refeições), com o aumento de problemas alimentares. Observou-se que a atitude excessivamente permissiva dos pais, a exemplo da pouca insistência para comer durante as refeições ou a frequente preparação de refeições especiais diferente dos demais membros da família, pode influenciar nos problemas alimentares das crianças. Assim, além de questões fisiológicas e sensoriais, fatores ligados às atitudes dos pais também podem interferir nos comportamentos alimentares do TEA. Outros estudos também descrevem a grande importância da dinâmica familiar na alimentação. Pais que apresentavam sobrepeso e possuíam problemas no controle da própria ingestão de alimentos (Birch \& Davison, 2001) influenciavam negativamente o hábito alimentar dos filhos (Hendrie, Sohonpal, 
Lange, \& Golley, 2013; Scaglioni, Arrizza, Vecchi, \& Tedeschi, 2011; Scaglioni, Salvioni, \& Galimberti, 2008). Outra contribuição do STEP-CHILD (Seiverling et al., 2011) foi a introdução do item "roubar comida" como outro aspecto comportamental do TEA e que anteriormente só havia sido descrito em adultos com necessidade especiais (Matson \& Kuhn, 2001). Algumas limitações devem ser salientadas no STEP-CHILD (Seiverling et al., 2011). Primeiramente não houve uma correlação significativa entre todos os fatores. Além disso, o tamanho da amostra foi pequeno, uma vez que das 142 crianças que participaram apenas 43 tinham TEA. A escala também não explorou de forma ampla os problemas sensoriais e gastrintestinais, os comportamentos ritualísticos e repetitivos característicos do transtorno, além de não ter avaliado os problemas alimentares em função da gravidade do TEA. $O$ artigo também não esclarece vários aspectos do estudo, a exemplo: a forma de recrutamento dos participantes, como foi realizada a confirmação do diagnóstico, se o grupo controle diferia dos demais, e não foram mencionadas avaliações relacionadas à estabilidade temporal da escala.

O SWEAA (Karlsson et al., 2013), apesar de ter demonstrado boa validade e confiabilidade interna baseada nos parâmetros estatísticos, não contemplou a heterogeneidade do transtorno. Ao estabelecerem um ponto de corte no valor do QI dos participantes, os pesquisadores criaram um grande fator limitante do estudo, e consequentemente a escala foi testada predominantemente $(72 \%)$ em autistas com alto rendimento com síndrome de Asperger diagnosticados anteriormente à publicação do DSM-V (American Psychiatric Association, 2013). Com relação aos fatores avaliados pelo conteúdo da escala, importantes tópicos ligados ao comportamento alimentar foram abordados nesse instrumento, a exemplo de controle motor, ambiente das refeições, comportamentos ritualísticos, aspectos sociais no momento das refeições e alguns distúrbios alimentares como o desejo de ingerir coisas que não são consideradas alimentos (pica). Também foram investigadas comorbidades associadas ao transtorno, a exemplo da depressão, do transtorno de déficit de atenção e hiperatividade (TDAH) e do transtorno obsessivo compulsivo (TOC). Contudo, os distúrbios gastrointestinais e a gravidade do TEA não foram contemplados. No processo de validação, também ocorreram alguns vieses: a adesão à pesquisa e a amostra foi pequena, pois apenas $28 \%$ dos indivíduos contatados participaram; o nível de escolaridade diferiu entre o grupo controle e intervenção; o QI do grupo com TEA não foi aferido, mas obtido dos registros médicos, e o do grupo controle não foi avaliado. Outras particularidades do SWEAA também são questionáveis, a exemplo de o ponto de corte na idade mínima ser de 15 anos. Os próprios autores também registraram como desvantagem o fato de o questionário ter sido de autorrelato e não haver ninguém disponível para auxiliar o entrevistado em caso de dúvidas. Segundo os autores, na análise do reteste da escala, uma possível limitação ocorreu porque a maioria dos participantes completou o questionário um ano antes do período previsto.

O BPFAS (Crist \& Napier-Phillips, 2001) é uma medida abrangente e amplamente utilizada para detectar problemas de alimentação e comportamentais baseadas em competências. É uma medida válida e confiável que discrimina de forma eficaz as 
crianças com problemas de alimentação em populações com desenvolvimento típico e com questões clínicas envolvidas. Mesmo sem ter passado por um devido processo de validação, o BPFAS (Crist \& Napier-Phillips, 2001) já havia sido utilizado em pesquisas envolvendo o TEA (Lukens \& Linscheid, 2008; Martins, Young, \& Robson, 2008) e em alguns casos como instrumento de avaliação do construto de outras escalas. Durante os procedimentos de validação e verificação estrutura do BPFAS em uma amostra com TEA (Allen et al., 2015), foi observada uma inadequação da estrutura de cinco fatores dessa escala, e os fatores "recusa de alimentos com textura" $(\alpha$ Cronbach $=0,26)$ e "recusa geral da criança mais velha" ( $\alpha$ Cronbach $=0,3$ ) demonstraram baixa consistência interna. O mesmo ocorreu em outros estudos nos quais a amostra de crianças possuía o diagnóstico de doença crônica ou estava com sobrepeso ou obesidade (Davis, Canter, Stough, Gillette, \& Patton, 2014). No que diz respeito à validação do construto do BPFAS, os pesquisadores observaram apenas correlações com pequeno a médio efeito. Nenhum dos três fatores que permaneceram após a análise fatorial demonstrou uma forte correlação com as variáveis investigadas. Apesar de ter sido desenvolvido para avaliar o problema alimentar de crianças de 1 a 8 anos de idade, o BPFAS (Crist \& Napier-Phillips, 2001) não levou em conta características específicas do TEA, como questões sensoriais e distúrbios gastrintestinais. Outro ponto a destacar é a estabilidade temporal que também não foi avaliada no estudo.

Existem dois aspectos comuns aos instrumentos de avaliação revisados: todos usaram o diagnóstico pelo DSM-IV no processo de validação do instrumento e nenhum considerou as queixas alimentares relatadas pelos pais. O DSM-IV trazia sob a epígrafe de transtornos invasivos do desenvolvimento o autismo, o transtorno desintegrativo da infância, a síndrome de Asperger, a síndrome de Rett e o transtorno invasivo do desenvolvimento sem outra especificação. O DMS-5, no entanto, reflete a visão científica de que esses transtornos (exceto a síndrome de Rett e o transtorno desintegrativo) são na verdade uma mesma condição, o TEA, com gravidade sintomática distinta em relação ao déficit na comunicação e interação social e ao padrão de comportamentos, interesses e atividades restritas e repetitivas (American Psychiatric Association, 2013). Assim, novos instrumentos para avaliar o comportamento alimentar de crianças com TEA devem contemplar os diferentes graus desse transtorno, conforme definidos no DSM-5. O outro aspecto comum a todos os instrumentos encontrados na literatura é que o ponto de partida para as questões formuladas é sempre a experiência clínica dos profissionais que elaboram a escala ou a literatura científica sobre o tema. Nenhum dos estudos aborda o que os pais de pessoas com TEA consideram como problemático no comportamento alimentar desses indivíduos. Assim, consideramos que existe a necessidade do desenvolvimento de um instrumento de avaliação do comportamento alimentar de pessoas com TEA que contemple essas duas necessidades. 


\section{Referências}

Abidin, R. R. (1995). Parenting stress index (3rd ed.). Odessa, FL: Psychological Assessment Resources.

Achenbach, T. M., \& Rescorla, L. A. (2000). Manual for ASEBA preschool forms \& profiles. Burlington, VT: University of Vermont, Research Center for Children, Youth \& Families.

Ahearn, W. H., Castine, T., Nault, K., \& Green, G. (2001). An assessment of food acceptance in children with autism or pervasive developmental disorder-not otherwise specified. Journal of Autism and Developmental Disorders, 31, 505-511. doi:10.1023/A:1012221026124

Allen, S. L., Smith, I. M., Duku, E., Vaillancourt, T., Szatmari, P., Bryson, S., Fombonne, E., Volden, J., Waddell, C., Zwaigenbaum, L., Roberts, W., Mirenda, P., Bennett, T., Elsabbagh, M., \& Georgiades, S. (2015). Behavioral Pediatrics Feeding Assessment Scale in young children with autism spectrum disorder: psychometrics and associations with child and parent variables. Journal of Pediatric Psychology, 40, 581-90. doi:10.1093/jpepsy/jsv006

American Psychiatric Association (2013). Diagnostic and statistical manual of mental disorders (DSM-V) (5th ed.) Washington, DC: American Psychiatric Association.

Bandini, L. G., Anderson, S. E., Curtin, C., Cermak, S., Evans, E. W., Scampini, R., Maslin, M., \& Must, A. (2010). Food selectivity in children with autism spectrum disorders and typically developing children. The Journal of Pediatrics, 157, 259-264. doi:10.1016/j.jpeds.2010.02.013

Birch, L. L., \& Davison, K. K. (2001). Family environmental factors influencing the developing behavioral controls of food intake and childhood overweight. Pediatric Clinics of North America, 48, 893-907. doi:10.1016/50031-3955(05)70347-3

Bodfish, J. W., Symons, F. J., Parker, D. E., \& Lewis, M. H. J. W. (2000). Varieties of repetitive behavior in autism: comparisons to mental retardation. Journal of Autism and Developmental Disorders, 30, 237-243. doi:10.1023/A:1005596502855

Call, N. A., Simmons, C. A., Mevers, J. E. L., \& Alvarez, J. P. (2015). Clinical outcomes of behavioral treatments for pica in children with development disabilities. Journal of Autism and Developmental Disorders, 45, 2105. doi:10.1007/s10803015-2375-z

Cermak, S. A., Curtin, C., \& Bandini, L. G. (2010). Food selectivity and sensory sensitivity in children with autism spectrum disorders. Journal of the American Dietetic Association, 110, 238-246. doi:10.1016/j.jada.2009.10.032

Chaidez, V., Hansen, R. L., \& Hertz-Picciotto, I. (2014). Gastrointestinal problems in children with autism, developmental delays or typical development. Journal of Autism and Developmental Disorders, 44, 1117-1127. doi:10.1007/s10803013-1973-x 
Constantino, J. N., \& Gruber, C. P. (2005). Social responsiveness scale. Los Angeles: Western Psychological Services.

Crist, W., \& Napier-Phillips, A. (2001). Mealtime behaviors of young children: a comparison of normative and clinical data. Journal of Developmental and Behavioral Pediatrics, 22, 279-286. doi:10.1097/00004703-200110000-00001

Davis, A. M., Canter, K. S., Stough, C. O., Gillette, M. D., \& Patton, S. (2014). Measurement of mealtime behaviors in rural overweight children: an exploratory factor analysis of the Behavioral Pediatrics Feeding Assessment Scale. Journal of Pediatric Psychology, 39, 332-339. doi:10.1093/jpepsy/jst089

Drewett, R. (2010). Psicologia nutricional da infância (S. Vieira, Trad.). Curitiba: Ibpex.

Gilliam, J. E. (1995). Gilliam autism rating scale: examiner's manual. Austin, TX: Pro-Ed.

Goodlin Jones, B. L., Sitnick, S. L., Tang, K., Liu, J., \& Anders, T. F. (2008). The Children's Sleep Habits Questionnaire in toddlers and preschool children. Journal of Developmental and Behavioral Pediatrics, 29, 82-88. doi:10.1097/DBP.0b013e$318163 c 39 a$

Green, S. A., \& Ben-Sasson, A. (2010). Anxiety disorders and sensory over-responsivity in children with autism spectrum disorders: is there a causal relationship? Journal of Autism and Developmental Disorders, 40, 1495-1504. doi:10.1007/s10 803-010-1007-x

Hendrie, G., Sohonpal, G., Lange, K., \& Golley, R. (2013). Change in the family food environment is associated with positive dietary change in children. International Journal of Behavioral Nutrition and Physical Activity, 10, 4-11. doi:10.1186/14795868-10-4

Hendy, H. M., Williams, K. E., Camise, T. S., Eckman, N., \& Hedemann, A. (2009). The Parent Mealtime Action Scale (PMAS) development and association with children's diet and weight. Appetite, 52, 328-339. doi:10.1016/j.appet.2008.11.003

Hyman, S. L., Stewart, P. A., Schmidt, B., Cain, U., Lemcke, N., Foley, J. T., Peck, R., Clemons, T., Reynolds, A., Johnson, C., Handen, B., James, S. J., Courtney, P. M., Molloy, C., \& Ng, P. K. (2012). Nutrient intake from food in children with autism. Pediatrics, 130, S145-153. doi:10.1542/peds.2012-0900L

Johnson, S. L. (2016). Developmental and environmental influences on young children's vegetable preferences and consumption. Advances in Nutrition, 7(1), 220S-231S. doi:10.3945/an.115.008706

Karlsson, L., Rastam, M., \& Went, E. (2013). The SWedish Eating Assessment for Autism spectrum disorders (SWEAA) - validation of a self-report questionnaire targeting eating disturbances within the autism spectrum. Research in Developmental Disabilities, 34, 2224-2233. doi:10.1016/j.ridd.2013.03.035 
Lockner, D. W., Crowe, T. K., \& Skipper, B. J. (2008). Dietary intake and parents' perception of mealtime behaviors in preschool-age children with autism spectrum disorder and in typically developing children. Journal of the American Dietetic Association, 108, 1360-1363. doi:10.1016/j.jada.2008.05.003

Lord, C., Risi, S., Lambrecht, L., Cook, E. H., Jr., Leventhal, B. L., Di Lavore, P. C., \& Rutter, M. (2000). The autism diagnostic observation schedule - generic: a standard measure of social and communication deficits associated with the spectrum of autism. Journal of Autism and Developmental Disorders, 30, 205-223. doi:10.10 23/A:1005592401947

Lukens, C. T. (2002). Assessing eating and mealtime behavior problems in children with autism. Unpublished master's thesis, The Ohio State University, Columbus, $\mathrm{OH}$.

Lukens, C. T., \& Linscheid, T. R. (2008). Development and validation of an inventory to assess mealtime behavior problems in children with autism. Journal of Autism and Developmental Disorders, 38, 342-352. doi:10.1007/s10803-007-0401-5

Mari-Bauset, S., Zazpe, I., Mari-Sanchis, A., Llopis-Gonzalez, A., \& Morales-SuarezVarela, M. (2013). Food selectivity in autism spectrum disorders: a systematic review. Journal of Child Neurology, 29, 1554-1561. doi:10.1177/0883073813498821

Martins, Y., Young, R. L., \& Robson, D. C. (2008). Feeding and eating behaviors in children with autism and typically developing children. Journal of Autism and Developmental Disorders, 38, 1878-1887. doi:10.1007/s10803-008-0583-5

Matson, J. L., \& Kuhn, D. E. (2001). Identifying feeding problems in mentally retarded persons: development and reliability of the screening tool of feeding problems (STEP). Research in Developmental Disabilities, 22, 165-172. doi:10.1016/ S0891-4222(01)00065-8

Novaes, C. M., Pondé, M. P., \& Freire, A. C. C. (2008). Control of psychomotor agitation and aggressive behavior in patients with autistic disorder: a retrospective chart review. Arquivos de Neuro-Psiquiatria, 66, 646-651. doi:10.1590/50004-282 X2008000500008

Owens, J. A., Spirito, A., \& McGuinn, M. (2000) The Children's Sleep Habits Questionnaire (CSHQ): psychometric properties of a survey instrument for school-aged children. Sleep, 23, 1043-1052. doi:10.1111/j.1746-1561.2009.00402.x

Rastam, M. (2008). Eating disturbances in autism spectrum disorders with focus on adolescent and adult years. Clinical Neuropsychiatry, 5, 31-42.

Rockett, H. R. H., Breitenbach, M., Frazier, A.L., Witschi, J., Wolf, A. M., Field, A. E., \& Colditz, G. A. (1997). Validation of a youth/adolescent food frequency questionnaire. Preventive Medicine, 26, 808-816. doi:10.1006/pmed.1997.0200

Rutter, M., Le Couteur, A., \& Lord, C. (2003). Autism diagnostic interview revised. Los Angeles, CA: Western Psychological Services. 
Sacrey, L. A., Germani, T., Bryson, S. E., \& Zwaigenbaum, L. (2014). Reaching and grasping in autism spectrum disorder: a review of recent literature. Frontiers in Neurology, 5, 1-12. doi:10.3389/fneur.2014.00006

Scaglioni, S., Arrizza, C., Vecchi, F., \& Tedeschi, S. (2011). Determinants of children's eating behavior. The American Journal of Clinical Nutrition, 94, 2006S-2011S. doi:10.3945/ajen.110.001685

Scaglioni, S., Salvioni, M., \& Galimberti, C. (2008) Influence of parental attitudes in the development of children eating behaviour. British Journal of Nutrition, 99, S22-S25. doi:10.1017/S0007114508892471.

Seiverling, L., Hendy, H. M., \& Williams, K. (2011). The Screening Tool of Feeding Problems applied to children (STEP-CHILD): psychometric characteristics and associations with child and parent variables. Research in Developmental Disabilities, 32, 1122-1129. doi:10.1016/j.ridd.2011.01.012

Sparrow, S. S., Cicchetti, D. V., \& Balla, D. A. (2005). Vineland adaptive behavior scales (2nd ed.). Livonia. MN: Pearson Assessments.

Wardle, J., Guthrie, C. A., Sanderson, S., \& Rapoport, L. (2001). Development of the children's eating behavior questionnaire. Journal of Child Psychology and Psychiatry, 42, 963-970. doi:10.1111/1469-7610.00792

Zimmerman, I., Steiner, V., \& Pond, R. (2002). Preschool language scale (4th ed.). San Antonio, TX: The Psychological Corporation.

Submissão: 28.9 .2017

Aceite: 4.6 .2018 